\title{
Effectiveness, quality and process evaluation of interventions to prevent childhood obesity.
}

\section{Abstract}

Childhood obesity is one of the most serious global public health challenges of the $21^{\text {st }}$ century. Health and scientific organizations demand early interventions, although their complexity generates difficulties in their implementation. In systematic reviews, quality assessments are intended to limit mislead reporting and it is important they are conducted in a manner that minimizes bias. Process evaluation (PE) is used to monitor and document program implementation and can aid in understanding the relationship between specific program elements and program outcomes. Failure to deliver an intervention as intended can lead to unclear conclusions about the effectiveness of the intervention. The aim of the present study is to evaluate the effectiveness of the studies based on the process evaluation and the quality on its interventions. We performed a systematic review of randomized control trials aiming to prevent childhood obesity. The Cochrane Handbook for Systematic Reviews of Interventions tool was used to assess and report methodological risk of bias. Each item was judged as being at high (HR), low (LR) or unclear (UN) risk of bias as per criteria. Key domains random sequence generation and allocation concealment were also assessed. Effectiveness was estimated when the study showed changes in body composition as the main outcome (BMI z-score or waist circumference). Process evaluation was evaluated if at least one process evaluation indicator was identified as being adequately implemented. From the 41 studies, 26 showed any degree of effectiveness and 15 were not effective. Almost half of studies scored high risk of bias: 20/41 (Effective 13/26; non effective 7/15). Moreover, 16/41 studies reported to have unclear risk of bias (effective 10/26; and non-effective 6/15). Only 5/41 articles reported to have low risk (effective 3/26; non effective 2/15). Regarding random sequence generation, there was no difference according to the quality of the studies (effective: LR 25/26, UN 1/26 AND HR 0/26; non effective: LR 15/15, UN 0/15 AND HR 0/15). Concerning the allocation concealment domain, there were no differences found either (effective: LR 11/26, UN 10/26 AND HR 5/26; non effective: LR 7/15, UN 5/15 AND HR 3/15). PE was used in 7 papers (effective 3/7 and non-effective 4/7). Quality seems to have slightly more influence in the non-effective studies (LR 13.3\%) than in the effective studies (LR 11.5\%). The non-effective studies showed the highest proportion of performing PE. There seems to be a relationship between the quality and PE performance.

\section{Funding information}

This project has received funding from the European Union's Horizon 2020 research and innovation programme under the Marie Sklowdowska-Curie grant agreement $\mathrm{N}^{\circ} 801586$. This project has also received funding from the University of Zaragoza-Mecenazgo Santander.

\section{Conflict of Interest}

There is no conflict of interest

*The original version of this article was published with missing Funding information. A notice detailing with this has been published and the error rectified in the online PDF and HTML copies. 